\title{
Stakeholder Engagement in Community-based Malaria Studies in a Defined Setting in the Eastern Province, Rwanda
}

\author{
Chantal Marie Ingabire, Msc ${ }^{1,2^{*}}$ \\ Fredrick Kateera, MD, MPH2,3 \\ Emmanuel Hakizimana, Msc 4,5 \\ Alexis Rulisa, MPH2,6 \\ Bart Van Den Borne, $\mathrm{PhD}^{2}$ \\ Claude Muvunyi, MD, PhD7 \\ Ingmar Nieuwold, Msc ${ }^{8}$ \\ Constantianus JM Koenraadt, $\mathrm{PhD}^{5}$ \\ Leon Mutesa, MD, $\mathrm{PhD}^{7}$ \\ Michele Van Vugt, MD, PhD³ \\ Jane Alaii, $\mathrm{PhD}^{9}$ \\ ${ }^{*}$ Corresponding author. Department of Health Promotion, Maastricht University, The Netherlands and \\ Medical Research Center Rwanda Biomedical Center, Kigali, Rwanda; Email: cingabire7@gmail.com \\ ${ }^{1}$ Medical Research Center, Rwanda Biomedical Center, Kigali, Rwanda \\ ${ }^{2}$ Department of Health Promotion, Maastricht University, The Netherlands \\ ${ }^{3}$ Academic Medical Center, Amsterdam, The Netherlands \\ ${ }^{4}$ Malaria \& Other Parasitic Diseases Division, Rwanda Biomedical Center, Kigali, Rwanda \\ ${ }^{5}$ Laboratory of Entomology, Wageningen University, Wageningen, The Netherlands \\ ${ }^{6}$ Department of Cultural Anthropology and Development Studies, Radboud University Nijmegen, Nijmegen, the Netherlands \\ ${ }^{7}$ College of Medicine and Health Sciences, University of Rwanda, Rwanda \\ ${ }^{8}$ Foundation The100th Village, Amsterdam, The Netherlands \\ ${ }^{9}$ Context Factor Solutions, Nairobi, Kenya
}

Doi:10.5901/mjss.2016.v7n2s1p188

\section{Abstract}

The value of engaging stakeholders for locally relevant responses and sustainable gains in disease control programs has been increasingly acknowledged. As opposed to a traditional top-down implementation of malaria intervention strategies, community bottom up initiatives require that all stakeholders be identified and strategies of engagement are designed at an early stage of program planning, implementation, and evaluation, to promote optimal intervention impact and program ownership and sustainability. A stakeholder analysis was conducted as part of a formative analysis in multiple community based studies under the malaria elimination program (MEPR) in Eastern Province of Rwanda. Starting with an initial list of stakeholders a snowball sampling technique was employed to identify other potential stakeholders from national and local public/private institutions and community/faith-based organizations. Individual interviews with nineteen stakeholders and eight focus group discussions with a total of 69 stakeholders were conducted. Stakeholders were classified into primary (lay community), secondary (local administrative and health institutions) and key stakeholders (policy makers and funders). Most of the stakeholders consulted were further classified depending on their type and degree of involvement unto information, consultation/collaboration, codecision and empowerment categories. The MEPR team independently assigned participatory communication techniques to stakeholders for further engagement. In addition to awareness about MEPR activities, stakeholders' reported willingness to contribute to the promotion of malaria preventive measures, participation in supportive hands-on trainings and in the MEPR planning (pre-engagement meetings and trainings), implementation (formative research and project interventions) and knowledge translation activities, such as the development of project materials as well as participation in lay and scientific workshops where research findings dissemination and interpretation were discussed. Overall, the analysis enabled the MEPR to know who to engage for a particular project activity and the appropriate time to do so. Stakeholders appreciated the early consultation by the MEPR and solicited continuous updates on malaria activities and key findings. Subsequently, stakeholder identification has been updated to evolve with shifting stakeholder interests over time.

Keywords: stakeholder analysis, community engagement, malaria, Rwanda. 


\section{Introduction}

Significant progress in the fight against malaria in Rwanda has been made as a result of the increased access to insecticide treated nets (LLINs) since 2004, indoor residual spraying (IRS) in malaria endemic districts since 2009 and the prompt treatment using artemisinin-based combination therapy (WHO, 2014). From 2005-2010, significant declines in malaria incidence (70\%), outpatient malaria cases (60\%) and inpatient malaria deaths (54\%) among children under five years have been reported (Karema et al., 2012). The maximization of the use of available preventive measures is important in a country whose national malaria strategic plan targets achievement of malaria pre-elimination levels by 2018 (Program, 2012).

Community participation at multiple levels is an important factor to achieve locally meaningful responses towards further malaria control and sustain the achieved gains (Atkinson, Vallely, Fitzgerald, Whittaker, \& Tanner, 2011). One approach towards sustainability of used interventions is effectively engaging with all stakeholders. To this end, a stakeholder analysis, a program-planning tool focused on identifying and analysing stakeholders' motivations for promoting or threatening malaria associated interventions, has been reported as necessary (Brugha \& Varvasovszky, 2000; Reed et al., 2009). Stakeholder analysis aims to understand stakeholder behaviour, intentions, interests and interrelations and to assess the influence and resources stakeholders may bring to decision making or implementation and analysis processes (Ancker \& Rechel, 2015; Freeman, 2001; Varvasovszky \& Brugha, 2000). As part of a social assessment, stakeholder analysis serves to identify key stakeholders and, based on their degree of involvement, establish an appropriate framework for participation in project selection, design, implementation, monitoring, and evaluation (Kansas, 2014; Luyet, Schlaepfer, Parlange, \& Buttler, 2012; Narayan, 1998; Prell, Hubacek, \& Reed, 2009; Reed et al., 2009; Reed, Stringer, Fazey, Evely, \& Kruijsen, 2014). In health care, stakeholder analysis is a tool that an organization can use to achieve specific advantages in collaborating with other institutions (Brugha \& Varvasovszky, 2000). Stakeholders with a common vision for the desired goals are an important strength for overcoming obstacles throughout program implementation (Njau, de Savigny, Gilson, Mwageni, \& Mosha, 2009).

A malaria elimination program (MEPR) was initiated in a community (Ruhuha sector) in the Eastern Province of Rwanda in 2012 (Ingabire et al., 2014) to complement the national malaria control program used interventions by stimulating the local community's active participation from an early stage in the planning, implementation and evaluation of local malaria activities. To achieve this, a stakeholder analysis was conducted to 1) identify local institutions and processes upon which to build further malaria control interventions, 2) be informed of stakeholders' participatory activities, (3) identify stakeholders' degree of involvement, and (4) determine the participatory and communication methods to be employed by the MEPR in engaging with stakeholders.

\section{Methods}

\subsection{Study site and sampling}

The MEPR activities are implemented in the moderate-low malaria transmission Ruhuha sector, Bugesera district, eastern province of Rwanda. The sector population is estimated to be 23893 individuals living in about 5098 households (Source: Ruhuha sector socio-economic categorization report, 2015).

Purposive sampling within and outside the Ruhuha sector was used to target all persons and groups with a stake in malaria control in Ruhuha sector and to ensure that a representative cross section of all relevant stakeholders were selected including members of district, regional and national malaria control efforts and those involved in designing and implementing malaria control policy (Hardon, 2001). Stakeholder identification was done using an iterative process. A preliminary list drawn from community representatives who participated in previous open space discussions that aimed at exploring different ways community can contribute towards malaria reduction towards elimination (Ingabire et al., 2014), was updated by MEPR team (PhD students) in close consultations with key informants from the National Malaria Control Program (NMCP), district health office, the local Ruhuha health centre and Ruhuha sector administrative office to generate a comprehensive stakeholder inventory. Based on the generated inventory list, a maximum variation sampling approach was used to purposively select a representative group of the entire cross section of stakeholders for interviews. A snowball technique was also employed where listed stakeholders were asked to identify additional potential stakeholders who may have considerable influence on MEPR activities including empowering the community towards eliminating malaria. With the exception of the community-level participants, the selection of all other respondents was conducted so as to purposely prioritise leaders in their respective organizations. Stakeholders were included from health, educational, religious, business and administrative sectors and also represented community-based organizations and the 
lay community. To generate a knowledge mapping, a checklist of open-ended questions (used during one-on-one interviews and group interviews) was developed to explore awareness of the MEPR and the stakeholder's proposed participatory actions towards malaria elimination.

\subsection{Data collection}

Interview appointments were made with all selected stakeholders. Initially, one-on-one followed by homogeneous group interviews were conducted as follows. Nineteen (19) stakeholders were interviewed in one-on-one interviews lasting approximately 30 minutes for each representative of public and private organizations such as non-governmental organizations (NGOs), churches, administrative and health professionals. Eight (8) group discussions (lasting approximately one hour each) were conducted with a total of sixty-nine (69) participants for homogeneous stakeholder groups of community health workers (CHWs) (10), members of local agricultural cooperatives (32), local school representatives (8), Ruhuha community members (10) and Ruhuha youth (9). All interviews and discussions were conducted in the local language "Kinyarwanda" by CMI (First author). Responses were recorded, transcribed and translated into English, coded and categorized for further comparison and analysis.

\subsection{Analysis}

We analysed our data using the analysis framework described elsewhere (Kansas, 2014; Luyet et al., 2012) in which four steps were followed. Firstly, interviewed individuals were categorized into primary (beneficiaries), secondary (involved with/or responsible for beneficiaries) and key (government officials, policy makers and donors) stakeholder groups. Secondly, analysis of stakeholders' knowledge about the MEPR programme and its activities followed by analysis of stakeholders' participatory actions related to malaria control and/or elimination was done. Thirdly, a description of stakeholders' degree of involvement was analysed in support of a model elaborated by Luyet et al. (Luyet et al., 2012), the degree of stakeholder involvement occurs on various levels and thus an illustration of this was guided by the authors' deliberation and judgment as previously recommended (Bryson, Cunningham, \& Lokkesmoe, 2002). The Luyet et al 's model (Luyet et al., 2012) describes five levels of analysis, however in our study, two levels were combined due to minor differences observed in our data to generate a 4-step model that includes: (1) information-explaining the project to the stakeholders; (2) consultation/collaboration-presenting the project to stakeholders, collecting their suggestions, and noting decision making with or without taking stakeholder input into account, (3) co-decisions-cooperating with stakeholders to achieve an agreement for solution and implementation, and (4) empowerment-delegating decisionmaking for project development and implementation to the stakeholders. The MEPR team established methods of communication for stakeholders and a further classification was made accordingly in the last step of analysis.

\section{Results}

\subsection{Stakeholder participation}

Eighty-eight (88) individual stakeholders were included in the analysis. The largest representation (32) was from locally based cooperatives group members. These cooperatives included stakeholders who worked in agriculture, transport, sewing, events decoration and security. The technical sector was made up of individuals from the NMCP, community health workers (CHWs) and local health centre staff, participants from community-based health insurance (CBHI) programs and representatives from the health district level, private clinics, drugstores and NGOs provided 22 participants. The community category included ten (10) adults and nine youth (age range of 21-35 years). Participants also included stakeholders from eight schools (three secondary schools, four primary schools and one vocational training school), five churches and two administrative-level staff from Ruhuha sector. Finally, stakeholders were classified into three categories depending on the nature of their participation (primary, secondary or key stakeholder) (table 1). The lay community was considered as the primary stakeholder, while 10 secondary stakeholders were identified, mostly from the health sector, but also from schools, churches and NGOs. Lastly, the health district office, Caritas Kigali (catholic organization that provides supervisory leadership to the Ruhuha health centre) and NMCP staffs were considered as key stakeholders. 
Table 1. Stakeholder Categorization

\begin{tabular}{clc}
\hline Primary stakeholders & Secondary stakeholders & Key stakeholders \\
\hline Lay community in the Ruhuha sector & Community health workers & Health district office staff \\
& Ruhuha health centre staff & NMCP staff Caritas Kigali \\
& Community-based health insurance staff & \\
& Administrative sector office staff & \\
& Drugstore staff \\
& Private clinic staff \\
Cooperative members & School staff \\
Church leaders & \\
NGOs staff & \\
\hline
\end{tabular}

\subsection{Awareness about MEPR}

Participants in both one-on-one and group sessions appeared almost universally aware of the MEPR organization and the activities being implemented in Ruhuha. Their awareness was mainly attributable to the prior active stakeholders' participation in the launch and follow-up of MEPR activities. Stakeholders including the Ruhuha health centre staff and CHWs were also directly involved in ongoing implementation of the MEPR including mobilizing households for project uptake and treating malaria cases identified during the project's conducted household baseline surveys.

\subsection{Participatory actions}

Based on the perceived seriousness of malaria in the area, stakeholders across all the interviews appeared in favour of the MEPR. Most stakeholders suggested partnering with the MEPR in community mobilization and sensitization for malaria preventive measures. On one hand, the health sector was more willing to assist the project with training community members to equip them with comprehensive malaria knowledge. On the other hand, stakeholders in this category suggested that the MEPR generates evidence that would be valuable to assess the impact of project interventions on the current malaria burden. The administrative sector was more interested in a mutual partnership with the health sector by mobilizing and sensitizing communities to increase the uptake of malaria preventive measures at this level. The business sector, general community members and youth preferred to focus on peer education and promoting a $\mathrm{CBHI}$ as important elements for prompt health care seeking. The private sector stakeholders, including staff from drugstores and private clinics acknowledged the need for prompt health-care seeking and a rational use of malaria medication based on confirmed cases, which suggests the establishment of a referral system in close collaboration with the local health centre. Lastly, the educational sector and youth suggested establishing anti-malaria clubs as a channel to share malaria-related information among the young generation in order to nurture pro-active action against malaria and trickling down to family members.

\subsection{Stakeholders by potential degree of involvement}

Of the 88 individuals, 75 were eligible for all steps of engagement. The first two steps (information and consultation/collaboration) were found to be more commonly reported as important for stakeholders. However, it was apparent that not every identified stakeholder was involved in all actions of the project as highlighted in table 2: the religious and education sector were not directly involved in the implementation of the MEPR, however were regularly informed, consulted and later on participated in the MEPR knowledge translation activities. 
Table 2. Degree of involvement in MEPR

\begin{tabular}{lcccc}
\hline Stakeholders & Information Consultation /Collaboration Co-decision Empowerment \\
Lay community & $*$ & $*$ & $*$ & $*$ \\
Business sector & $*$ & $*$ & $*$ & $*$ \\
Religious sector & $*$ & $*$ & & \\
Education sector & $*$ & $*$ & $*$ & $*$ \\
Health sector & $*$ & $*$ & $*$ & $*$ \\
Administrative sector & $*$ & $*$ & & \\
\hline
\end{tabular}

\subsection{Stakeholder participatory methods for communication}

Following identification, categorization of stakeholders and highlighting their degrees of involvement and identifying their potential contributory actions towards MEPR activities, the study team established communication methods to gather data, share information and/or identify key research and malaria control related activities for further collaboration (table 3). All stakeholders participated in the data collection processes, either through open space discussions, annually conducted surveys or individual or group interviews. Some of the stakeholders participated in the MEPR implementation and were therefore provided with hands-on trainings to equip them with knowledge and practical skills needed to perform their activities. Phone messaging was used as a reminder for regular meetings and monthly progress reports. All stakeholders were invited to attend meetings where study findings were presented and follow up discussions on presented findings were held. In addition, study findings and key MEPR activities were printed and distributed using brochures. Lastly, virtual stakeholders were targeted using peer-reviewed publications, policy briefs as well as online blogs and documentaries.

Table 3. Communication/engagement methods by stakeholder

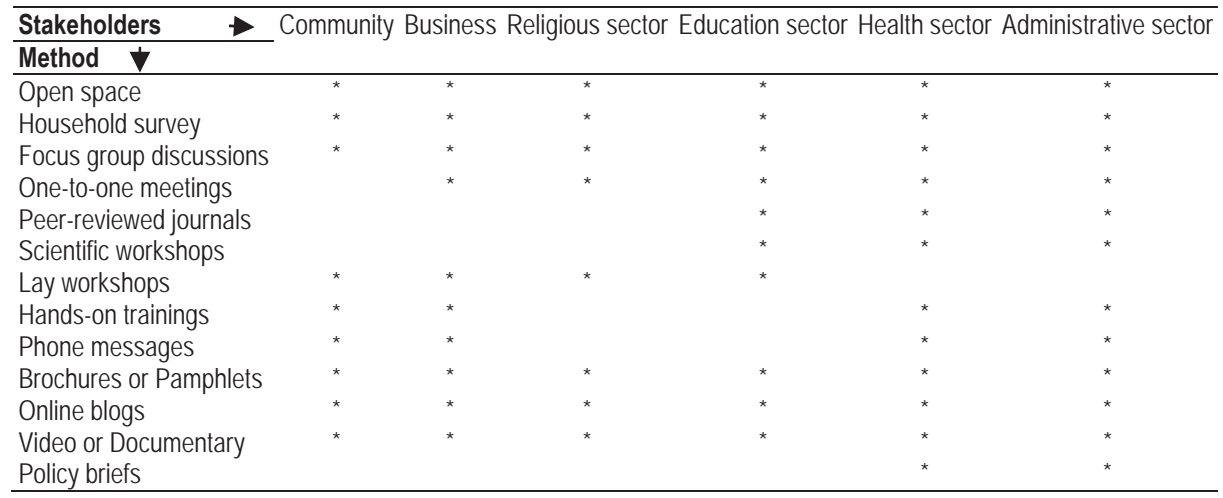

\subsection{Application of stakeholder analysis: insights into MEPR}

The MEPR deploys a bottom-up approach towards maximizing the use of available preventive malaria control tools to further reduce the local malarial burden and seeks to adapt its goals to the needs and priorities of likely users (Reed et al., 2014; Thomas \& Palfrey, 1996). Two elements of engagement as described by Pretty (1995) were conceptually applied in this analysis. The first element was self-mobilization emerging from community initiatives and stakeholders making key decisions in regular meetings (Pretty, 1995). Based on this principle, some identified stakeholders were further grouped in a platform called community malaria-action teams (CMATs) to collectively identify local malaria-related problems and adopt solutions to remedy these problems. The platforms also served as liaisons between the MEPR project and the community at large and facilitated knowledge exchange among members and MEPR team through feedback sessions that were set up to promote CMATs and MEPR project activities implementation. Within a year of being established (2013-2014), CMATs were found to have largely contributed to the MEPR' s goal of further malaria control by facilitating community preparedness for planned MEPR research activities and promoting active community mobilization towards the use of available malaria preventive measures such as the use of LLINs, acceptance of IRS, 
clearing peridomestic potential mosquito breeding sites and supporting community member's health care seeking behaviours. A reported significant reduction in presumed malaria/fever cases from $68 \%$ to $21.4 \%$ and an increase in health insurance coverage from $66.3 \%$ to 91\% were observed among others (Household surveys 2013 and 2014). Furthermore, on-going community sensitization contributed to an increase in community acceptance of IRS, which led to 100\% coverage in early 2015 compared to $91 \%$ coverage reported in 2014.

The second element of engagement was interaction- the provision of greater opportunities for stakeholders to be involved in decision making and consideration of stakeholders as a means of achieving predetermined project objectives (Pretty, 1995). Thus, the MEPR team further engaged identified stakeholders from rice cooperatives in a mosquito-larval source management program in the irrigated rice fields that harbour the malaria mosquito larvae. These stakeholders reported that being involved in the planning stage contributed to an increase in their level of ownership and knowledge based on reflective learning.

Effective knowledge exchange has been highlighted as an important aspect to enhance the impact on policy and practice and foster positive relationships (McInnes et al., 2012; Reed et al., 2014). In this regard, the MEPR researchers held a series of results-dissemination and interpretation meetings as part of the stakeholder engagement strategy. The audiences for the series included policymakers and the scientific and lay communities to create awareness on the MEPR findings and to highlight future provisions.

Although, the initial analysis did not involve stakeholders from the media sector, the contribution of media became apparent during the results dissemination and thus their engagement was prioritized.

MEPR researchers also interviewed stakeholders with regard to their expertise and based on their reported willingness to participate in funding MEPR project activities and supporting the packaging of research findings for knowledge translation. With this, hands -on trainings on how to engage various stakeholders in the field using online blogs, brochures, documentaries, policy briefs and dialogues were held in collaboration with one of the stakeholders with an extensive expertise in knowledge translation. This led to a positive partnership between MEPR team and their network of stakeholders as well as the stakeholders' actions that led to increased awareness about MEPR activities and findings, better sensitization of Ruhuha community members about on-going malaria elimination efforts, and promoted the sharing of best practices with all stakeholders.

\section{Discussion}

Input from a wide range of stakeholders is essential for developing a participatory, consensus-building process that meets the needs and expectations of both the community and program implementers. Our initial interviews provided a substantive list of stakeholders, across various categories, and identified the support they could potentially provide to the MEPR activities. A large number of stakeholders expressed their willingness to provide technical support in relation to the project implementation as well as active participation in malaria related educational activities. The local administrative office expressed particular interest in providing political support to facilitated project acceptance and engagement in MEPR related activities at the community level.

Many stakeholders actively participated in implementation of project interventions, which fostered a sense of reflective learning and ownership in malaria elimination efforts.

With financial and technical support provided to the MEPR by one of its stakeholders for research-related knowledge translation activities, packaging of study findings from research conducted was noted as essential to increasing awareness and subsequent use of research findings beyond the scope of participating stakeholders.

Our study findings suggest that all primary and secondary stakeholders appeared strongly interested in the MEPR as a result of being directly affected by malaria. However, due to high levels of interdependency between stakeholders, successful change requires a close partnership with the three stakeholder categories; primary, secondary, and key stakeholder categories to achieve a greater impact.

The innovative idea of establishing malaria clubs among school children and youth at large aligns well with what has been previously reported in the same community and underscores the importance of this idea for further malaria control (Ingabire et al., 2014; Ingabire et al., 2015). The MEPR project has thus far informed and consulted the educational sector in regards to proper implementation of activities. Future collaboration may require the creation of such clubs and exploration of their impact in reversing malaria burden, specifically among young generation.

The systematic identification of stakeholders in a research process has been reported as an effective way to increase the value and likelihood of community engagement (Reed et al., 2014), especially when tackling an issue recognized as a priority to the stakeholder audience and hence participation requires that all interests and/or concerns of stakeholders are addressed (Mallery C, 2012; Namazzi et al., 2013). The multi stakeholder-based approach in the early 
stage of the project facilitated MEPR team's knowledge of who, when and how to engage and thus resulted in the support and agreement of malaria control related interventions and processes as also suggested elsewhere (Hyder et al., 2010; Namazzi et al., 2013; Reed et al., 2009).

Previous experience has shown that communication content and processes within research settings are influenced by the socio-cultural environment. Thus adapting approaches based on cognizance of the context enhances wider access to relevant information for various levels of stakeholders (Hyder et al., 2010). In our study, identification of communication channels between MEPR and stakeholders was key importance for planning and implementation of MEPR project activities and served as platform to share and/or receive tailored messages for both project team and stakeholders.

Active participation of stakeholders was eminent throughout in the conducted MEPR activities. In contrast in Uganda, where a study evaluating the role and influence of actors in malaria treatment policy making reported the lay community as non-participatory actors, rather considered by policy makers as beneficiaries (Nabyonga-Orem, Nanyunja, Marchal, Criel, \& Ssengooba, 2014). This Ugandan study recommended availability of structures and systems to enable community participation in both research process and decision-making(Nabyonga-Orem et al., 2014).

Despite the ability of stakeholder analysis to predict and generate information, the results of this analysis are amenable change as influenced by stakeholders' interests, position, leadership and other attributes and thus requires continued close monitoring (Brugha \& Varvasovszky, 2000). To maintain constant engagement of stakeholders and relevance of the analysis throughout the duration of research, it has been important for MEPR facilitators to manage the conduct and content of stakeholder engagement by tailoring support and maintaining contact with stakeholders through meetings or workshops as observed elsewhere (Brugha \& Varvasovszky, 2000; Concannon et al., 2014). For this reason, the MEPR operationalized a bi-directional communication and feedback strategy. Annually, research results dissemination and interpretation workshops with community, scientific, funders and policy makers were organized to validate the findings, discuss implications and propose a way forward. In addition, quarterly meetings for hands-on trainings and discussions on action plan with primary stakeholders, mostly through CMAT members were organized to implement malaria related prevention activities in close collaboration with the community. Lastly, regular formal and informal meetings with secondary stakeholders, mostly local administrative and health offices for proper planning and execution of project activities were planned and implemented. Similarly to other settings, these one-to-one meetings were reported to foster relationships and promote understanding of research priorities and clarify specific stakeholder roles (Christen O'Haire, 2011). As a result, Ruhuha area where MEPR activities are being implemented, reported positive changes such as the reduction of presumed malaria cases, increase of health insurance coverage as well as complete household coverage of IRS. The MEPR partnership with relevant stakeholders has therefore contributed to the capacity building of the local community in terms of knowledge and skills while accelerating the achievement of the main project goal of malaria elimination through community-based and context-tailored approaches.

The combination of individual and group interviews was preferred considering that creating social conditions for heterogeneous groups is often challenging (Jinks, Ong, \& O'Neill, 2009). This resulted in gathering evidence for stakeholder identification, categorization and engagement. The fact that some stakeholders were already linked with the project was not necessarily regarded as a limitation to the analysis but as positively contributory to the perceived awareness on MEPR activities and purpose among area stakeholders.

This paper illustrates the steps taken to identify and engage relevant stakeholders in the MEPR related activities. Further studies focusing on a detailed evaluation of the overall stakeholder contribution to the main goal of malaria reduction moving towards elimination, highlighting some of the pitfalls and generating recommendations towards a sustainable stakeholder engagement are recommended.

Findings from this analysis should be interpreted in view of some limitations as some of the interviews were conducted with individuals from institutions or organizations while individuals may not necessarily represent the views of the institutions from which they represented.

\section{Conclusion}

In our study, a stakeholder-focused approach enabled identification of multiple stakeholders from different levels of administrative units and lay community; definition of their role as well as their degree of involvement in planning and implementation of an effective community-based malaria elimination project in Ruhuha sector, Rwanda. Stakeholder identification was inclusive of all relevant individuals and organizations and the analysis was performed at an early stage and updated to evolve with shifting stakeholder interests over time. Appropriate analysis in relation to stakeholder expertise was considered so the MEPR could learn who to engage for a particular project activity and the appropriate time to do so. This process highlighted the value of stakeholder engagement in ensuring sustainability, ownership and collaboration for optimal impact of malaria control programs in a defined community. 


\section{References}

Ancker, S., \& Rechel, B. (2015). HIVIAIDS policy-making in Kyrgyzstan: a stakeholder analysis. Health Policy Plan, 30(1), 8-18. doi:10.1093/heapol/czt092

Atkinson, J.-A., Vallely, A., Fitzgerald, L., Whittaker, M., \& Tanner, M. (2011). The architecture and effect of participation: a systematic review of community participation for communicable disease control and elimination. Implications for malaria elimination. Malar $J$, 10(1), 225. Retrieved from http://www.malariajournal.com/content/10/1/225

Brugha, R., \& Varvasovszky, Z. (2000). Stakeholder analysis: a review. Health Policy and Planning, 15(3), 239-246. doi:10.1093/heapol/ 15.3.239

Bryson, J. M., Cunningham, G. L., \& Lokkesmoe, K. J. (2002). What to Do When Stakeholders Matter: The Case of Problem Formulation for the African American Men Project of Hennepin County, Minnesota. Public Administration Review, 62(5), 568-584. doi:10.2307/3110017

Christen O'Haire, M. M., Erika Nakamoto, Lia LaBrant, Carole Most, Kathy Lee, Elaine Graham, Erika Cottrell, Jeanne-Marie Guise. (2011). Methods for Engaging Stakeholders To Identify and Prioritize Future Research Needs. . Retrieved from Rockville: http://www.effectivehealthcare.ahrq.gov/ehc/products/200/698/MFRNGuide04--Engaging_Stakeholders--6-10-2011.pdf

Concannon, T. W., Fuster, M., Saunders, T., Patel, K., Wong, J. B., Leslie, L. K., \& Lau, J. (2014). A systematic review of stakeholder engagement in comparative effectiveness and patient-centered outcomes research. J Gen Intern Med, 29(12), 1692-1701. doi:10.1007/s11606-014-2878-x

Freeman, R. E. a. M., John,. (2001). A Stakeholder Approach to Strategic Management doi:http://dx.doi.org/10.2139/ssrn.263511

Hardon, A., Boonmongkon, P., Streefland, P., Tan, L. (2001). Applied Health Research Manual: Anthropology of Health and Health Care: New Jersey: Transaction Publishers.

Hyder, A., Syed, S., Puvanachandra, P., Bloom, G., Sundaram, S., Mahmood, S., ... Peters, D. (2010). Stakeholder analysis for health research: case studies from low- and middle-income countries. Public Health, 124(3), 159-166. doi:10.1016/j.puhe.2009.12.006

Ingabire, C., Alaii, J., Hakizimana, E., Kateera, F., Muhimuzi, D., Nieuwold, I., .. Van Den Borne, B. (2014). Community mobilization for malaria elimination: application of an open space methodology in Ruhuha sector, Rwanda. Malar J, 13(1), 167. Retrieved from http://www.malariajournal.com/content/13/1/167

Ingabire, C., Rulisa, A., Van Kempen, L., Muvunyi, C., Koenraadt, C., Van Vugt, M., ... Alaii, J. (2015). Factors impeding the acceptability and use of malaria preventive measures: implications for malaria elimination in eastern Rwanda. Malar J, 14(1), 136. Retrieved from http://www.malariajournal.com/content/14/1/136

Jinks, C., Ong, B., \& O'Neill, T. (2009). The Keele community knee pain forum: action research to engage with stakeholders about the prevention of knee pain and disability. BMC Musculoskeletal Disorders, 10(1), 85. Retrieved from http://www.biomedcentral.com/ $1471-2474 / 10 / 85$

Kansas, U. 0. (2014). Identifying and Analyzing Stakeholders and Their Interests Retrieved from http://ctb.ku.edu/en/table-ofcontents/participation/encouraging-involvement/identify-stakeholders/main. http://ctb.ku.edu/en/table-of-contents/participation/ encouraging-involvement/identify-stakeholders/main

Karema, C., Aregawi, M., Rukundo, A., Kabayiza, A., Mulindahabi, M., Fall, I., . . Binagwaho, A. (2012). Trends in malaria cases, hospital admissions and deaths following scale-up of anti-malarial interventions, 2000-2010, Rwanda. Malar J, 11(1), 236. Retrieved from http://www.malariajournal.com/content/11/1/236

Luyet, V., Schlaepfer, R., Parlange, M. B., \& Buttler, A. (2012). A framework to implement Stakeholder participation in environmental projects. Journal of Environmental Management, 111(0), 213-219. doi:http://dx.doi.org/10.1016/j.jenvman.2012.06.026

Mallery C, G. D., Fernandez J, Smeeding L, Robinson S, Moon M, Lavallee D, Siegel J. (2012). Innovative Methods in Stakeholder Engagement: An Environmental Scan. Retrieved from http://www.effectivehealthcare.ahrq.gov/tasks/sites/ehc/assets/File/CF_ Innovation-in-Stakeholder-Engagement_LiteratureReview.pdf

McInnes, E., Middleton, S., Gardner, G., Haines, M., Haertsch, M., Paul, C., \& Castaldi, P. (2012). A qualitative study of stakeholder views of the conditions for and outcomes of successful clinical networks. BMC Health Services Research, 12(1), 49. Retrieved from http://www.biomedcentral.com/1472-6963/12/49

Nabyonga-Orem, J., Nanyunja, M., Marchal, B., Criel, B., \& Ssengooba, F. (2014). The roles and influence of actors in the uptake of evidence: the case of malaria treatment policy change in Uganda. Implement Sci, 9, 150. doi:10.1186/s13012-014-0150-8

Namazzi, G., N, K., Peter, W., John, B., Olico, O., A, A., . . . Elizabeth, E. (2013). Stakeholder analysis for a maternal and newborn health project in Eastern Uganda. BMC Pregnancy and Childbirth, 13(1), 58. Retrieved from http://www.biomedcentral.com/ $1471-2393 / 13 / 58$

Narayan, J. R.-M. a. D. (1998). Participation and Social Assessment: Tools and Techniques. Library of Congress Cataloging-inPublication Data.

Njau, R. J., de Savigny, D., Gilson, L., Mwageni, E., \& Mosha, F. W. (2009). Implementation of an insecticide-treated net subsidy scheme under a public-private partnership for malaria control in Tanzania-challenges in implementation. Malaria Journal, 8, 201. doi:10.1186/1475-2875-8-201

Prell, C., Hubacek, K., \& Reed, M. (2009). Stakeholder Analysis and Social Network Analysis in Natural Resource Management. Society \& Natural Resources, 22(6), 501-518. doi:10.1080/08941920802199202

Pretty, J. N. (1995). Participatory learning for sustainable agriculture. World Development, 23(8), 1247-1263. doi:http://dx.doi.org/10.10 16/0305-750X(95)00046-F 
Program, R. N. M. C. (2012). Rwanda Malaria Control Strategic Plan July 2013- June 2018.

Reed, M. S., Graves, A., Dandy, N., Posthumus, H., Hubacek, K., Morris, J., . . Stringer, L. C. (2009). Who's in and why? A typology of stakeholder analysis methods for natural resource management. J Environ Manage, 90(5), 1933-1949. doi:10.1016/j.jenvman. 2009.01.001

Reed, M. S., Stringer, L. C., Fazey, I., Evely, A. C., \& Kruijsen, J. H. J. (2014). Five principles for the practice of knowledge exchange in environmental management. Journal of Environmental Management, 146(0), 337-345. doi:http://dx.doi.org/10.1016/j.jenvman. 2014.07.021

Thomas, P., \& Palfrey, C. (1996). Evaluation: Stakeholder-focused Criteria. Social Policy \& Administration, 30(2), 125-142. doi:10.1111/j.1467-9515.1996.tb00432.x

Varvasovszky, Z., \& Brugha, R. (2000). A stakeholder analysis. Health Policy and Planning, 15(3), 338-345. doi:10.1093/heapol/15.3.338 WHO. (2014). World Malaria Report 2014. 